\title{
A New Approach to Proinov-Type Fixed-Point Results in Non-Archimedean Fuzzy Metric Spaces
}

\author{
Mi Zhou ${ }^{1, *(\mathbb{D})}$, Naeem Saleem ${ }^{2}(\mathbb{D})$, Xiaolan Liu ${ }^{3,4,5, * \mathbb{D}}$, Andreea Fulga ${ }^{6}(\mathbb{D}$ \\ and Antonio Francisco Roldán López de Hierro ${ }^{7, *}$ (D)
}

1 School of Science and Technology, University of Sanya, Sanya 572022, China

2 Department of Mathematics, University of Management and Technology, Lahore 54770, Punjab, Pakistan; naeem.saleem2@gmail.com

3 College of Mathematics and Statistics, Sichuan University of Science and Engineering, Zigong 643000, China

4 Key Laboratory of Higher Education of Sichuan Province for Enterprise Informationlization and Internet of Things, Zigong 643000, China

5 South Sichuan Center for Applied Mathematics, Zigong 643000, China

6 Department of Mathematics and Computer Science, Transilvania University of Brasov, 500036 Brasov, Romania; afulga@unitbv.ro

7 Department of Statistics and Operations Research, University of Granada, 18071 Granada, Spain

* Correspondence: mizhou@sanyau.edu.cn (M.Z.); xiaolanliu@suse.edu.cn (X.L.); aroldan@ugr.es (A.F.R.L.d.H.)

check for

updates

Citation: Zhou, M.; Saleem, N.; Liu, X.; Fulga, A.; Roldán López de Hierro, A.F. A New Approach to

Proinov-Type Fixed-Point Results in

Non-Archimedean Fuzzy Metric

Spaces. Mathematics 2021, 9, 3001.

https: / / doi.org/

10.3390/math9233001

Academic Editor: Christopher Goodrich

Received: 20 October 2021

Accepted: 17 November 2021

Published: 23 November 2021

Publisher's Note: MDPI stays neutral with regard to jurisdictional claims in published maps and institutional affiliations.

\begin{abstract}
Very recently, by considering a self-mapping $T$ on a complete metric space satisfying a general contractivity condition of the form $\psi(d(T x, T y)) \leq \varphi(d(x, y))$, Proinov proved some fixedpoint theorems, which extended and unified many existing results in the literature. Accordingly, inspired by Proinov-type contraction conditions, Roldán López de Hierro et al. introduced a novel family of contractions in fuzzy metric spaces (in the sense of George and Veeramani), whose main advantage is the very weak constraints imposed on the auxiliary functions that appear in the contractivity condition. They also proved the existence and uniqueness of fixed points for the discussed family of fuzzy contractions in the setting of non-Archimedean fuzzy metric spaces. In this paper, we introduce a new family of fuzzy contractions based on Proinov-type contractions for which the involved auxiliary functions are not supposed to satisfy any monotonicity assumptions; further, we establish some new results about the existence and uniqueness of fixed points. Furthermore, we show how the main results in the above-mentioned paper can be deduced from our main statements. In this way, our conclusions provide a positive partial solution to one of the open problems posed by such authors for deleting or weakening the hypothesis of the nondecreasingness character of the auxiliary functions.
\end{abstract}

Keywords: fuzzy metric space; fixed point; Proinov-type contraction; non-Archimedean fuzzy metric space

MSC: 47H10; 47H09; 54H25; 46T99

\section{Introduction}

Fixed-point theory has become one of the most attractive fields in nonlinear analysis and even mathematics in general, due to its ability to find solutions of nonlinear equations, such as functional equations, matrix equations [1-4], integral equations [5-7], etc. Therefore, it is an essential and powerful tool for solving some existence problems because of its wide applications in areas such as computer science, engineering, economics, physics, game theory and many other fields. It is well known that after Banach's pioneering statement [8] in 1922, thousands of results that generalize or extend the famous Banach contraction principle have appeared. Among those remarkable results, given an arbitrary self-operator, two main concerns must be considered: an appropriate generalized contractive condition, and 
a reasonable abstract metric structure of the discussed space. A contractivity condition usually guarantees that the distance between the images through the operator $T$ of two distinct points of the space is lower than or equal to the distance of two such points, and also that the Picard iterative sequence $\left\{T^{n} x_{0}\right\}$ converges to the fixed point of $T$ for any initial point $x_{0}$.

Very recently, Proinov introduced in [9] a great family of contractions to propose some novel metric fixed point theorems that cover many earlier fixed point results, including the attractive results presented by Wardowski [10], and Jleli and Samet [11]. He also showed that some recently presented results are actually equivalent to the special cases of Skof's theorem [12]. The reason why Proinov-type contractions have attracted the attention of many researchers is due to the fact that these contractions involve a wide class of auxiliary functions satisfying some very weak constraints. Consequently, a variety of contractive conditions that inherit or improve the property of Proinov-type contractions has encouraged many mathematicians to persist in the study of this class of contractions (see [13-15]).

On the other hand, the second main development direction of fixed-point theory, above mentioned, is to study more general metric structures on the underlying spaces. A kind of significant extension of the family of metric spaces is called fuzzy metric spaces. In 1965, Zadeh [16] introduced the concept of a fuzzy set. Since then, one of the important problems in this field of study has been to obtain an adequate notion of fuzzy metric space. There are several ways to introduce the notion of metric in the fuzzy setting. After the approaches due to Menger [17] (statistical metric spaces), Kaleva and Seikkala [18], Schweizer and Sklar [19] (probabilistic metric spaces), Kramosil and Michálek [20] (fuzzy metric spaces), and others (see [21]), George and Veeramani [22] introduced a wide class of fuzzy metric spaces which further has demonstrated to be special, according to the needs of fixed-point theory (see, for instance, [21-28]). Moreover, to overcome some shortcomings of the notion of fuzzy metric spaces in the study of fixed-point theory, an additional assumption is often introduced: the non-Archimedean property. This property establishes that the same real parameter can relate the fuzzy distance between any three points of the underlying space. This property is very useful in practice because the main examples of fuzzy metric spaces that are handled in applications satisfy such a constraint.

Inspired by Proinov's results, in [14], the authors introduced a new class of contractions in the setting of fuzzy metric spaces (in the sense of George and Veeramani) and proved some fixed point results that improved some previous theorems by using a very general class of restrictions on the involved auxiliary functions. Motivated by the contributions of $[9,14]$, in this paper, we introduce a novel family of contractions based on the Proinovtype contractions for which the involved auxiliary functions are supposed to satisfy weaker constraints, and we describe some new results about the existence of unique fixed points in non-Archimedean fuzzy metric spaces. Furthermore, we prove that the main results in [14] can be deduced from our main results. Simultaneously, our conclusions provide a positive partial solution to one of the open problems posed in [14] for deleting or weakening the hypothesis of nondecreasingness on auxiliary functions.

\section{Preliminaries}

For the sake of convenience and completeness, we briefly recall some basic concepts and preliminaries to be used henceforth. Let $\mathbb{R}$ and $\mathbb{N}$ be the families of all real numbers and all positive integers, respectively.

Proposition 1. If $f:(0,1] \rightarrow \mathbb{R}$ is a function and $\left\{t_{n}\right\} \subset(0,1]$ is a nondecreasing sequence such that $f\left(t_{n}\right) \rightarrow+\infty$, then there is $\alpha \in(0,1]$ and a partial subsequence $\left\{t_{n_{k}}\right\}_{k \in \mathbb{N}}$ of $\left\{t_{n}\right\}_{n \in \mathbb{N}}$ such that the following holds:

$$
t_{n_{k}}<t_{n_{k+1}}<\alpha \text { for all } k \in \mathbb{N}, \quad t_{n_{k}} \rightarrow \alpha \text { and } f\left(t_{n_{k}}\right) \rightarrow+\infty \text { as } k \rightarrow \infty .
$$


Proof. Since $\left\{t_{n}\right\}$ is nondecreasing and bounded from above, it is convergent. Let $\alpha \in(0,1]$ be its limit, that is, assume that $t_{n} \rightarrow \alpha$ and $t_{n} \leq t_{n+1} \leq \alpha$ for all $n \in \mathbb{N}$. If there is $n_{0} \in \mathbb{N}$ such that $t_{n_{0}}=\alpha$, then $\alpha=t_{n_{0}} \leq t_{n} \leq \alpha$ for all $n \geq n_{0}$, that is, $t_{n}=\alpha$ for all $n \geq n_{0}$. However, this is impossible because $f\left(t_{n}\right) \rightarrow+\infty$. Therefore, $t_{n}<\alpha$ for all $n \in \mathbb{N}$. In such a case, the sequence $\left\{t_{n}\right\}$ has an strictly increasing partial subsequence $\left\{t_{n_{k}}\right\}$ such that $t_{n_{k}}<t_{n_{k+1}}<\alpha$ for all $k \in \mathbb{N}$. As it is a partial subsequence of $\left\{t_{n}\right\}$, we conclude that $t_{n_{k}} \rightarrow \alpha$ and $f\left(t_{n_{k}}\right) \rightarrow+\infty$ as $k \rightarrow \infty$.

Let $T: X \rightarrow X$ be a map from a nonempty set $X$ into itself. If a point $x_{0} \in X$ satisfies $T x_{0}=x_{0}$, then $x_{0}$ is a fixed point of $T$. We denote by Fix $(T)$ the set of all fixed points of $T$.

A sequence $\left\{x_{n}\right\}$ in $X$ is almost periodic if there is $n_{0}, k_{0} \in \mathbb{N}$ such that $x_{n+k_{0}}=x_{n}$ for all $n \geq n_{0}$. A sequence $\left\{x_{n}\right\}$ in $X$ is infinite if $x_{m} \neq x_{n}$ for all $m \neq n$. A sequence $\left\{x_{n}\right\} \subseteq X$ is called a Picard sequence of $T$ based on $x_{0} \in X$ if $x_{n+1}=T x_{n}$ for all $n \in \mathbb{N} \cup\{0\}$. Notice that in such a case, $x_{n}=T^{n} x_{0}$ for each $n \in \mathbb{N} \cup\{0\}$, where $\left\{T^{n}: X \rightarrow X\right\}$ are the iterates of $T$ defined by $T^{0}=$ identity, $T^{1}=T$ and $T^{n+1}=T \circ T^{n}$ for $n \geq 1$.

Definition 1. A binary operation $*:[0,1] \times[0,1] \rightarrow[0,1]$ is called a continuous $t$-norm if it satisfies the following assertions:

$(T 1) *$ is commutative and associative.

(T2) $*$ is continuous.

(T3) $a * 1=a$, for all $a \in[0,1]$.

(T4) $a * b \leq c * d$ whenever $a \leq c$ and $b \leq d$ for all $a, b, c, d \in[0,1]$.

Some classical examples of continuous t-norms are stated as follows.

- $\quad$ Product $*_{p}: \quad a *_{p} b=a \cdot b$.

- $\quad$ Minimum $*_{m}: \quad a *_{m} b=\min \{a, b\}$.

- $\quad$ Łukasiewicz $*_{L}: \quad a *_{L} b=\max \{0, a+b-1\}$.

Definition 2 ([29], Definition 4). A t-norm is continuous at 1-boundary if it is continuous at each point of type $(1, s)$, where $s \in[0,1]$ (that is, if $t_{n} \rightarrow 1$ and $s_{n} \rightarrow s$, then $t_{n} * s_{n} \rightarrow 1 * s=s$.)

Definition 3. A fuzzy metric space in the sense of George and Veeramani is an ordered triple $(X, M, *)$ such that $X$ is a nonempty set, $*$ is a continuous $t$-norm and $M$ is a fuzzy set on $X \times X \times(0,+\infty)$ satisfying the following conditions for all $x, y, z \in X$ and $t, s>0$ :

$\left(F_{1}\right) M(x, y, t)>0$.

$\left(F_{2}\right) M(x, y, t)=1$ for all $t>0$ if and only if $x=y$.

$\left(F_{3}\right) M(x, y, t)=M(y, x, t)$.

$\left(F_{4}\right) M(x, y, t) * M(y, z, s) \leq M(x, z, t+s)$.

$\left(F_{5}\right) M(x, y, \cdot):(0,+\infty) \rightarrow[0,1]$ is continuous.

Then, the triple $(X, M, *)$ is called a fuzzy metric space. If we replace $\left(F_{4}\right)$ by $\left(F_{6}\right),\left(F_{6}\right)$ $M(x, z, t) \geq M(x, y, t) * M(y, z, t)$ or $M(x, z, \max \{t, s\}) \geq M(x, y, t) * M(y, z, s)$, then the triple $(X, M, *)$ is called a non-Archimedean fuzzy metric space.

Since $\left(F_{6}\right)$ implies $\left(F_{4}\right)$, then each non-Archimedean fuzzy metric space is a fuzzy metric space.

For the sake of generalization, we will only assume that the t-norm is continuous at the 1-boundary.

Lemma 1 ([30], Lemma 2.5). If $(X, M, *)$ is a fuzzy metric space, then $M(x, y, \cdot)$ is nondecreasing on $(0,+\infty)$ for all $x, y \in X$.

Example 1. Let $X=\mathbb{R}$. Define $a * b=a \cdot b$ and the following:

$$
M(x, y, t)=\exp \left(-\frac{|x-y|}{t}\right),
$$


for all $x, y \in X$ and all $t>0$. Then, $(X, M, *)$ is a fuzzy metric space.

Example 2. Let $(X, d)$ be a metric space. Define $a * b=a \cdot b$ and, given $k, m, n \in \mathbb{R}^{+}$, the following:

$$
M(x, y, t)=\frac{k t^{n}}{k t^{n}+m d(x, y)}
$$

for all $x, y \in X$ and all $t>0$. Then, $(X, M, *)$ is a fuzzy metric space. Taking $k=n=m=1$ in the above example, we have the following:

$$
M(x, y, t)=\frac{t}{t+d(x, y)} .
$$

We call this fuzzy metric, induced by a metric $d$, the standard fuzzy metric.

Example 3 ([23], Example 1.3). Let $(X, d)$ be a metric space and let $\vartheta$ be a nondecreasing and continuous function from $(0,+\infty)$ into $(0,1)$ such that $\lim _{t \rightarrow+\infty} \vartheta(t)=1$. Let $*$ be a $t$-norm such that $* \leq *_{p}$. For each $x, y \in X$ and $t>0$, define the following:

$$
M(x, y, *)=(\vartheta(t))^{d(x, y)} .
$$

Then $(X, M, *)$ is a non-Archimedean fuzzy metric space.

Definition 4. Let $(X, M, *)$ be a fuzzy metric space. Then, we have the following:

(i) Sequence $\left\{x_{n}\right\} M$-converges to $x \in X$ if $\lim _{n \rightarrow+\infty} M\left(x_{n}, x, t\right)=1$ for all $t>0$;

(ii) Sequence $\left\{x_{n}\right\}$ in $X$ is an $M$-Cauchy sequence if for all $\epsilon \in(0,1)$ and $t>0$, there exists $n_{0} \in \mathbb{N}$ such that $M\left(x_{m}, x_{n}, t\right)>1-\epsilon$ for all $m, n \geq n_{0}$;

(iii) The fuzzy metric space is called $M$-complete if every M-Cauchy sequence converges to some $x \in X$.

Proposition 2 ([14], Proposition 2). Let $\left\{x_{n}\right\}$ be a Picard sequence in a fuzzy metric space $(X, M, *)$ such that $M\left(x_{n}, x_{n+1}, t\right) \rightarrow 1$ for all $t>0$. If there are $m_{0}, n_{0} \in \mathbb{N}$ such that $m_{0}<n_{0}$ and $x_{m_{0}}=x_{n_{0}}$, then there is $l_{0} \in \mathbb{N}$ and $x^{*} \in X$ such that $x_{n}=x^{*}$ for all $n \geq l_{0}$ (that is, $\left\{x_{n}\right\}$ is constant from a term onward). In such a case, $x^{*}$ is a fixed point of the self-mapping for which $\left\{x_{n}\right\}$ is a Picard sequence.

Proposition 3. Every Picard sequence is either infinite or almost periodic.

Definition 5 ([29], Definition 21). We say that a fuzzy space (X, M) satisfies the property $\mathcal{N C}$ ("not Cauchy") if for each sequence $\left\{x_{n}\right\} \subseteq X$, which is not M-Cauchy and verifies $\lim _{n \rightarrow+\infty} M\left(x_{n}, x_{n+1}, t\right)=1$ for all $t>0$, there are $\varepsilon_{0} \in(0,1)$ and $t_{0}>0$ and two partial subsequences $\left\{x_{m_{k}}\right\}$ and $\left\{x_{n_{k}}\right\}$ of $\left\{x_{n}\right\}$ such that for all $k \in \mathbb{N}$, the following holds:

$$
\begin{aligned}
& k<m_{k}<n_{k}<m_{k+1} \text { and } \\
& M\left(x_{m_{k}}, x_{n_{k}-1}, t_{0}\right)>1-\varepsilon_{0} \geq M\left(x_{m_{k}}, x_{n_{k}}, t_{0}\right), \\
& \lim _{n \rightarrow+\infty} M\left(x_{m_{k}}, x_{n_{k}}, t_{0}\right)=\lim _{n \rightarrow+\infty} M\left(x_{m_{k}-1}, x_{n_{k}-1}, t_{0}\right)=1-\varepsilon_{0} .
\end{aligned}
$$

Very recently, Proinov [9] considered a self-mapping $T$ on a complete metric space satisfying a very general contractivity condition of the form $\psi(d(T x, T y)) \leq \varphi(d(x, y))$, and proved some fixed point theorems which extend and unify many earlier results in the literature. 
Theorem 1 ([9], Theorem 3.6). Let $(X, d)$ be a complete metric space and $T: X \rightarrow X$ be a mapping such that

$$
\psi(d(T x, T y)) \leq \varphi(d(x, y))
$$

for all $x, y \in X$ with $d(T x, T y)>0$, where the functions $\varphi, \psi:(0,+\infty) \rightarrow \mathbb{R}$ satisfy the following conditions:

(i) $\psi$ is nondecreasing.

(ii) $\varphi(s)<\psi(s)$ for any $s>0$.

(iii) $\lim _{s \rightarrow \epsilon^{+}} \sup \varphi(s)<\psi(\epsilon+)$ for any $\epsilon>0$.

Then $T$ has a unique fixed point $x_{0} \in X$ and the iterative sequence $\left\{T^{n} x_{0}\right\}$ converges to $x^{*}$ for every $x_{0} \in X$.

Inspired by the above theorem, the authors in [14] considered the Proinov-type contractivity condition defined by the following inequality:

$$
\varphi(M(T u, T v, s)) \geq \eta(M(u, v, s)),
$$

for all $u, v \in X$ with $T u \neq T v$ and all $s>0$, where the pair $(\varphi, \eta)$ belongs to a new family of auxiliary functions, which is illustrated in the following definition.

Definition 6 ([14], Definition 4). We denote by $\mathcal{L}$ the family of pairs $(\varphi, \eta)$ of functions $\varphi, \eta$ : $(0,1] \rightarrow \mathbb{R}$ verifying the following properties:

$\left(p_{1}\right) \varphi$ is nondecreasing.

$\left(p_{2}\right) \eta(s)>\varphi(s)$ for any $s \in(0,1)$.

$\left(p_{3}\right) \liminf _{s \rightarrow L^{-}} \eta(s)>\lim _{s \rightarrow L^{-}} \varphi(s)$ for any $L \in(0,1)$.

$\left(p_{4}\right)$ if $t \in(0,1]$ is such that $\varphi(t) \geq \eta(1)$, then $t=1$.

Here are some examples of pairs of functions belonging to $\mathcal{L}$ :

(1) $\varphi(s)=s$ and $\eta(s)=\sqrt{s}$, for all $s \in(0,1]$.

(2) $\varphi(s)=s^{2}$ and $\eta(s)=s$, for all $s \in(0,1]$.

The next theorem presented in [14] describes sufficient conditions in order to ensure that a self-mapping admits a unique fixed point in the setting of fuzzy metric spaces, satisfying the non-Archimedean assumption.

Theorem 2 ([14], Theorem 2). Let $(X, M, *)$ be an M-complete non-Archimedean fuzzy metric space and let $T: X \rightarrow X$ be a mapping for which there exists $(\varphi, \eta) \in \mathcal{L}$ such that

$$
\varphi(M(T u, T v, s)) \geq \eta(M(u, v, s)),
$$

for all $u, v \in X$ with $T u \neq T v$ and all $s>0$.

Then each iterative Picard sequence $\left\{T^{n} u\right\}$ converges to the unique fixed point of $T$ for every initial condition $u \in X$.

The Proinov contractivity condition is very distinct in nature to its fuzzy version mainly due to the fact that the fuzzy metric spaces include an additional variable $s \in$ $(0,+\infty)$ for modeling the ambiguity about the exact value of the distance between two points. Hence, the involved auxiliary functions that appear in both contraction conditions have to satisfy very different conditions. In the first case, Proinov showed in [9] that it is possible to develop the metric fixed point theory when $\psi$ and $\varphi$ satisfy conditions $(i)$-(iii) described in Theorem 1. Accordingly, assumptions $\left(p_{1}\right)-\left(p_{4}\right)$ have also proved to be able to deal with the corresponding problem in the fuzzy setting. However, the monotone 
conditions " $(i) \psi$ is nondecreasing" and " $\left(p_{1}\right) \varphi$ is nondecreasing" severely restrict the family of functions that can be used in this field of study. As a consequence, very recently, some authors have posed this question as an open problem in the framework of fixed-point theory.

In the coming section, we provide a novel family of auxiliary functions that can be employed to handle contraction conditions while enjoying the advantage that it generalizes the family of functions given in [14] by avoiding the monotone condition, which is replaced by a more algebraic property. Moreover, the assumption of monotonicity on auxiliary functions is removed in our main results, which gives a positive partial solution to Open Problem 2 in [14]: can the hypothesis of nondecreasingness be removed (or replaced by a weaker assumption) from Theorem 2 ?

\section{The Contractive Condition and a Class of Auxiliary Functions}

We start this section by introducing a new family of auxiliary functions as follows. Let $\varphi, \eta:(0,1] \rightarrow \mathbb{R}$ be two functions satisfying the following properties:

$\left(C_{1}\right)$ for every $1>r \geq t>0$, one has $\eta(r)>\varphi(t)$;

$\left(C_{2}\right) \lim _{s \rightarrow L^{-}} \eta(s)>\limsup _{s \rightarrow L^{-}} \varphi(s)$ for each $L \in(0,1)$;

$\left(C_{3}\right)$ if $t \in(0,1]$ such that $\varphi(t) \geq \eta(1)$, then $t=1$.

We shall denote by $\mathcal{F}$ the family of all pairs of mappings $(\varphi, \eta)$ that satisfy the conditions $\left(C_{1}\right)-\left(C_{3}\right)$. It is easy to check that this family is nonempty, even considering non-continuous functions. The following ones are some examples of pairs $(\varphi, \eta)$ belonging to $\mathcal{F}$ :

- $\varphi(s)=s$ and $\eta(s)=\sqrt{s}$ for all $s \in(0,1]$.

- $\varphi(s)=s^{2}$ and $\eta(s)=s$ for all $s \in(0,1]$.

- $\varphi(s)=s^{3}$ and $\eta(s)=\sqrt{s}$ for all $s \in(0,1]$.

- $\varphi(s)=s$ and $\eta(s)=1-\lambda(1-s)$ for all $s \in(0,1]$ and $\lambda \in(0,1)$.

- $\varphi(s)=\left\{\begin{array}{ll}s, & s \in\left(0, \frac{1}{2}\right), \\ s^{3}, & s \in\left[\frac{1}{2}, 1\right],\end{array}\right.$ and $\eta(s)= \begin{cases}2 s, & s \in\left(0, \frac{1}{2}\right), \\ 1, & s \in\left[\frac{1}{2}, 1\right] .\end{cases}$

Proposition 4. The condition $\left(C_{2}\right)$ implies the condition $\left(C_{2}^{\prime}\right)$ stated as follows:

$$
\left(C_{2}^{\prime}\right): \quad \liminf _{s \rightarrow L^{-}}(\eta(s)-\varphi(s))>0 \text { for any } L \in(0,1) .
$$

Proof. Suppose that $\left(C_{2}\right)$ holds but $\left(C_{2}^{\prime}\right)$ is false. Then, there is $L_{0} \in(0,1)$ such that the following holds:

$$
\liminf _{s \rightarrow L_{0}^{-}}(\eta(s)-\varphi(s)) \leq 0
$$

Let us define $\delta_{0}=-\liminf _{s \rightarrow L_{0}^{-}}(\eta(s)-\varphi(s)) \geq 0$, that is, we are assuming the following:

$$
\liminf _{s \rightarrow L_{0}^{-}}(\eta(s)-\varphi(s))=-\delta_{0} \leq 0
$$

As this limit inferior is $-\delta_{0}$, then there exists a sequence $\left\{s_{n}\right\}_{n \in \mathbb{N}} \subset\left(0, L_{0}\right)$ such that the following holds:

$$
\begin{aligned}
& s_{n}<s_{n+1}<L_{0} \text { for all } n \in \mathbb{N}, \\
& s_{n} \rightarrow L_{0} \text { and } \\
& \lim _{n \rightarrow \infty}\left(\eta\left(s_{n}\right)-\varphi\left(s_{n}\right)\right)=-\delta_{0} \leq 0 .
\end{aligned}
$$


Since $s_{n} \rightarrow L_{0}^{-}$and $\left(C_{2}\right)$ hold, then the limit $\lim _{s \rightarrow L_{0}^{-}} \eta(s)$ exists, and it is equal to the following:

$$
\lim _{s \rightarrow L_{0}^{-}} \eta(s)=\lim _{n \rightarrow \infty} \eta\left(s_{n}\right) .
$$

Then by taking limits in the following expression,

$$
\varphi\left(s_{n}\right)=\eta\left(s_{n}\right)-\left(\eta\left(s_{n}\right)-\varphi\left(s_{n}\right)\right),
$$

we deduce the following:

$$
\begin{aligned}
\lim _{n \rightarrow \infty} \varphi\left(s_{n}\right) & =\lim _{n \rightarrow \infty}\left[\eta\left(s_{n}\right)-\left(\eta\left(s_{n}\right)-\varphi\left(s_{n}\right)\right)\right] \\
& =\lim _{n \rightarrow \infty} \eta\left(s_{n}\right)-\lim _{n \rightarrow \infty}\left(\eta\left(s_{n}\right)-\varphi\left(s_{n}\right)\right) \\
& =\lim _{s \rightarrow L_{0}^{-}} \eta(s)-\left(-\delta_{0}\right) \\
& =\lim _{s \rightarrow L_{0}^{-}} \eta(s)+\delta_{0} .
\end{aligned}
$$

As a result, we have the following:

$$
\limsup _{s \rightarrow L_{0}^{-}} \varphi(s) \geq \lim _{n \rightarrow \infty} \varphi\left(s_{n}\right) \geq \lim _{s \rightarrow L_{0}^{-}} \eta(s)+\delta_{0} \geq \lim _{s \rightarrow L_{0}^{-}} \eta(s),
$$

which contradicts the condition $\left(C_{2}\right)$.

Now, we show that the condition $\left(C_{2}^{\prime}\right)$ can be equivalently stated in an alternative way by using series of non-negative terms.

Lemma 2. Let $\varphi, \eta:(0,1] \rightarrow \mathbb{R}$ be two functions satisfying the following:

$\left(p_{2}\right) \eta(s)>\varphi(s)$ for any $s \in(0,1)$.

Then, the following conditions are equivalent:

$\left(C_{2}^{\prime}\right) \liminf _{s \rightarrow L^{-}}(\eta(s)-\varphi(s))>0$ for any $L \in(0,1)$.

$\left(C_{2}^{\prime \prime}\right)$ For each nondecreasing sequence $\left\{t_{n}\right\} \subset(0,1)$ such that $t_{n} \rightarrow L \in(0,1)$ the series of positive terms $\sum_{n \geq 1}\left(\eta\left(t_{n}\right)-\varphi\left(t_{n}\right)\right)$ diverges.

$\left(C_{2}^{\prime \prime \prime}\right)$ For each strictly increasing sequence $\left\{t_{n}\right\} \subset(0,1)$ such that $t_{n} \rightarrow L \in(0,1)$ the series of positive terms $\sum_{n \geq 1}\left(\eta\left(t_{n}\right)-\varphi\left(t_{n}\right)\right)$ diverges.

Proof. $\left[\left(C_{2}^{\prime}\right) \Rightarrow\left(C_{2}^{\prime \prime}\right)\right]$ Let $\left\{t_{n}\right\} \subset(0,1)$ be a nondecreasing sequence such that $t_{n} \rightarrow$ $L \in(0,1)$. Consider the real number

$$
\varepsilon_{0}=\liminf _{s \rightarrow L^{-}}(\eta(s)-\varphi(s))>0,
$$

which is strictly positive by $\left(C_{2}\right)$. Therefore,

$$
0<\varepsilon_{0}<\liminf _{s \rightarrow L^{-}}(\eta(s)-\varphi(s)) \leq \liminf _{n \rightarrow+\infty}\left(\eta\left(t_{n}\right)-\varphi\left(t_{n}\right)\right) .
$$

Hence, the series of positive terms $\sum_{n \geq 1}\left(\eta\left(t_{n}\right)-\varphi\left(t_{n}\right)\right)$ diverges.

$\left[\left(C_{2}^{\prime \prime}\right) \Rightarrow\left(C_{2}^{\prime \prime \prime}\right)\right]$ It is apparent.

$\left[\left(C_{2}^{\prime \prime \prime}\right) \Rightarrow\left(C_{2}^{\prime}\right)\right]$ Reasoning by contradiction, suppose that there exists $L \in(0,1)$ such that the following holds:

$$
\liminf _{s \rightarrow L^{-}}(\eta(s)-\varphi(s))=0 .
$$


Then one can find a sequence $\left\{t_{n}\right\} \subset(0,1)$ such that the following holds:

$$
t_{n} \rightarrow L, \quad t_{n}<L \quad \text { for all } n \in \mathbb{N}, \quad \text { and } \quad \liminf _{n \rightarrow+\infty}\left(\eta\left(t_{n}\right)-\varphi\left(t_{n}\right)\right)=0 .
$$

Without loss of generality, we assume that $\left\{t_{n}\right\}$ is strictly increasing. Then there exists $n_{1} \in \mathbb{N}$ such that the following holds:

$$
\eta\left(t_{n_{1}}\right)-\varphi\left(t_{n_{1}}\right)<\frac{1}{2}
$$

Similarly, we can also find $n_{2}>n_{1}$ such that the following holds:

$$
\eta\left(t_{n_{2}}\right)-\varphi\left(t_{n_{2}}\right)<\frac{1}{2^{2}}
$$

By induction, we can find a partial subsequence $\left\{t_{n_{k}}\right\}$ of $\left\{t_{n}\right\}$ such that the following holds:

$$
\eta\left(t_{n_{k}}\right)-\varphi\left(t_{n_{k}}\right)<\frac{1}{2^{k}} \quad \text { for all } k \geq 1 .
$$

Thus, the series $\sum_{k>1}\left(\eta\left(t_{n_{k}}\right)-\varphi\left(t_{n_{k}}\right)\right)$ converges and $t_{n_{k}} \rightarrow L$ as $k \rightarrow+\infty$. This contradicts the condition $\left(C_{2}^{\prime \prime \prime}\right)$.

Corollary 1. If we replace the condition $\left(p_{2}\right)$ in Lemma 2 by the following,

$\left(C_{1}\right)$ For every $1>r \geq t>0$, one has $\eta(r)>\varphi(t)$,

then Lemma 2 remains true.

Proof. It follows from the fact that $\left(C_{1}\right)$ implies $\left(p_{2}\right)$ (use $r=t$ ).

Next, we introduce a novel property in order to ensure that fixed point theory is able to be developed under these conditions.

Definition 7. A function $f:(0,1] \rightarrow \mathbb{R}$ satisfies the property $\left(P^{*}\right)$ when the following condition holds:

$\left(P^{*}\right)$ If there is a nondecreasing sequence $\left\{t_{n}\right\} \subset(0,1]$ such that $f\left(t_{n}\right) \rightarrow+\infty$, then $t_{n} \rightarrow 1$.

We must clarify that when there is no nondecreasing sequence $\left\{t_{n}\right\} \subset(0,1]$ such that $f\left(t_{n}\right) \rightarrow+\infty$, we accept that the function $f$ satisfies the property $\left(P^{*}\right)$. Such a condition can be stated in a more convenient way for proving some results.

Proposition 5. A function $f:(0,1] \rightarrow \mathbb{R}$ satisfies the property $\left(P^{*}\right)$ if, and only if, the following holds:

- If there exists a sequence $\left\{t_{n}\right\} \subset(0,1]$ converging to $\alpha \in(0,1]$ such that $t_{n}<t_{n+1}<\alpha$ for all $n \in \mathbb{N}$ and $f\left(t_{n}\right) \rightarrow+\infty$, then $\alpha=1$.

Proof. The condition is clearly necessary. To prove that it is also sufficient, suppose that there is a nondecreasing sequence $\left\{t_{n}\right\} \subset(0,1]$ such that $f\left(t_{n}\right) \rightarrow+\infty$. By Proposition 1 , there is $\alpha \in(0,1]$ and a partial subsequence $\left\{t_{n_{k}}\right\}_{k \in \mathbb{N}}$ of $\left\{t_{n}\right\}_{n \in \mathbb{N}}$ such that the following holds:

$$
t_{n_{k}}<t_{n_{k+1}}<\alpha \quad \text { for all } k \in \mathbb{N}, \quad t_{n_{k}} \rightarrow \alpha \quad \text { and } \quad f\left(t_{n_{k}}\right) \rightarrow+\infty \quad \text { as } k \rightarrow \infty .
$$

Using the assumption, we deduce that $\alpha=1$, so $t_{n} \rightarrow \alpha=1$ and $f$ satisfies the property $\left(P^{*}\right)$. 
In the next result, we show some examples of functions satisfying the property $\left(P^{*}\right)$.

Lemma 3. Let $f:(0,1] \rightarrow \mathbb{R}$ be a function verifying at least one of the following conditions:

1. $f$ is bounded from above.

2. $f$ is nondecreasing.

3. $f$ is left-continuous.

4. $f$ is upper semi-continuous.

5. $f$ is continuous.

Then, there does not exist any nondecreasing sequence $\left\{t_{n}\right\} \subset(0,1]$ such that $f\left(t_{n}\right) \rightarrow+\infty$. As a consequence, $f$ satisfies the property $\left(P^{*}\right)$.

Proof. In the first two cases, it is impossible that there is a sequence $\left\{t_{n}\right\} \subset(0,1]$ such that $f\left(t_{n}\right) \rightarrow+\infty$ because $f$ is bounded from above (in the second case, $f(t) \leq f(1)$ for all $t \in(0,1])$. Therefore, $f$ satisfies the property $\left(P^{*}\right)$.

Next, we assume by contradiction. Suppose that there is a nondecreasing sequence $\left\{t_{n}\right\} \subset$ $(0,1]$ such that $f\left(t_{n}\right) \rightarrow+\infty$. By Proposition 1 , there is $\alpha \in(0,1]$ and a partial subsequence $\left\{t_{n_{k}}\right\}_{k \in \mathbb{N}}$ of $\left\{t_{n}\right\}_{n \in \mathbb{N}}$ such that the following holds:

$$
t_{n_{k}}<t_{n_{k+1}}<\alpha \text { for all } k \in \mathbb{N}, \quad t_{n_{k}} \rightarrow \alpha \text { and } f\left(t_{n_{k}}\right) \rightarrow+\infty \text { as } k \rightarrow \infty .
$$

Case 3. If $f$ is left-continuous, then the following holds:

$$
\lim _{k \rightarrow+\infty} f\left(t_{n_{k}}\right)=f\left(\lim _{k \rightarrow+\infty} t_{n_{k}}\right)=f(\alpha) \in \mathbb{R},
$$

which contradicts that $f\left(t_{n_{k}}\right) \rightarrow+\infty$.

Case 4. Since $f$ is upper semi-continuous at $t=\alpha$, associated to $\varepsilon_{0}>0$, there is $\delta_{0}>0$ such that the following holds:

$$
f(t) \leq f(\alpha)+\varepsilon_{0} \quad \text { for each } t \in(0,1] \text { such that }|t-\alpha|<\delta_{0} .
$$

Since $t_{n_{k}} \rightarrow \alpha$ as $k \rightarrow \infty$, there exists $k_{1} \geq k_{0}$ such that $t_{n_{k}} \in(0,1]$ and $\left|t_{n_{k}}-\alpha\right|<\delta_{0}$ for all $k \geq k_{1}$. Therefore $f\left(t_{n_{k}}\right) \leq f(\alpha)+\varepsilon_{0}$ for all $k \geq k_{1}$, which contradicts $f\left(t_{n_{k}}\right) \rightarrow+\infty$.

Case 5. The proof is straightforward from the fact that continuity implies upper semi-continuity.

Corollary 2. If $(\varphi, \eta)$ is a pair of functions belonging to $\mathcal{L}$, then the function $\varphi$ satisfies the property $\left(P^{*}\right)$.

Proof. We have the following from Lemma 3, taking into account that $\varphi$ is nondecreasing.

Note that Lemma 3 shows some classes of functions satisfying the property $\left(P^{*}\right)$. However, there exist functions satisfying the property $\left(P^{*}\right)$, which do not satisfy any of the conditions of Lemma 3, as we show in the following result.

Proposition 6. Let $\left\{r_{n}\right\}$ be a strictly increasing sequence in $(0,1]$ converging to 1 and let $F, G$ : $(0,1] \rightarrow \mathbb{R}$ be two functions such that $\lim _{n \rightarrow+\infty} F\left(r_{n}\right)=+\infty$ and $G$ is bounded from above. Then the function $f:(0,1] \rightarrow \mathbb{R}$ given by

$$
f(t)= \begin{cases}F(t), & t \in\left\{r_{n}: n=1,2, \ldots\right\}, \\ G(t), & t \notin\left\{r_{n}: n=1,2, \ldots\right\},\end{cases}
$$


satisfies the property $\left(P^{*}\right)$. Furthermore, if $\Delta_{f}$ and $\Delta_{G}$ denote the sets of discontinuity points of $f$ and $G$, respectively, then the following holds:

$$
\Delta_{f} \subseteq\left\{r_{n}: n \in \mathbb{N}\right\} \cup \Delta_{G} .
$$

In addition to this, the function $f$ does not satisfy any of the conditions of Lemma 2.

Proof. To prove that $f$ satisfies the property $\left(P^{*}\right)$, we use the characterization given by Proposition 5. Suppose that there is a sequence $\left\{t_{n}\right\} \subset(0,1]$ converging to $\alpha \in(0,1]$ such that $t_{n}<t_{n+1}<\alpha$ for all $n \in \mathbb{N}$ and $f\left(t_{n}\right) \rightarrow+\infty$. Since $G$ is bounded from above, there is $R \in \mathbb{R}$ such that $G(t) \leq R$ for all $t \in(0,1]$. Taking into account that $f\left(t_{n}\right) \rightarrow+\infty$, there is $n_{0} \in \mathbb{N}$ such that $f\left(t_{n}\right)>R$ for all $n \geq n_{0}$. Hence, $t_{n} \in\left\{r_{k}: k \in \mathbb{N}\right\}$ for all $n \geq n_{0}$, that is, $t_{n}=r_{k_{n}}$ for all $n \geq n_{0}$. As $\left\{t_{n}\right\} \rightarrow \alpha$ and $t_{n}<t_{n+1}$ for all $n \in \mathbb{N}$, then $\left\{r_{k_{n}}\right\}_{n \in \mathbb{N}}$ converges to $\alpha$. However, as $\left\{r_{k_{n}}\right\}$ is a partial subsequence of $\left\{r_{n}\right\}$, and $r_{n} \rightarrow 1$, then the following holds:

$$
\alpha=\lim _{n \rightarrow+\infty} t_{n}=\lim _{n \rightarrow+\infty} r_{k_{n}}=1 .
$$

Therefore, $t_{n} \rightarrow 1$ and $f$ satisfies the property $\left(P^{*}\right)$. The second part follows from the fact that $\left.f\right|_{\left(r_{n}, r_{n+1}\right)}=\left.G\right|_{\left(r_{n}, r_{n+1}\right)}$ for all $n \in \mathbb{N}$, so $f$ can be discontinuous at $\left\{r_{n}: n \in \mathbb{N}\right\}$ and also at the points where $G$ is discontinuous.

Finally, the function $f$ does not satisfy any of the conditions of Lemma 2:

- It is not bounded from above because $f\left(t_{n}\right) \rightarrow+\infty$;

- It is not nondecreasing because if $n \geq n_{0}$, then

$$
r_{n}<\frac{r_{n}+r_{n+1}}{2} \text { but } f\left(\frac{r_{n}+r_{n+1}}{2}\right)=G\left(\frac{r_{n}+r_{n+1}}{2}\right) \leq R<f\left(r_{n}\right) ;
$$

- $f$ does not verify any kind of continuity at the points $\left\{r_{n+1}: n \geq n_{0}\right\}$ because for $n \geq$ $n_{0}$ and $t \in\left(r_{n}, r_{n+1}\right) \cup\left(r_{n+1}, r_{n+2}\right)$, we have $f(t)=G(t) \leq R<F\left(r_{n+1}\right)=f\left(r_{n+1}\right)$.

This completes the proof.

The previous result permits to introduce a great variety of functions satisfying the property $\left(P^{*}\right)$ that do not verify any of the conditions of Lemma 2.

Example 4. If the sequence $\left\{r_{n}\right\}$ is given by $r_{n}=1-\frac{1}{2 n+1}$ for $n \in \mathbb{N}$ and $f:(0,1] \rightarrow \mathbb{R}$ is the function defined by the following:

$$
f(t)= \begin{cases}\tan \left(\frac{\pi}{2} t\right), & \text { if } t \in\left\{r_{n}: n=1,2, \ldots\right\}, \\ t, & \text { if } t \notin\left\{r_{n}: n=1,2, \ldots\right\},\end{cases}
$$

and then $f$ satisfies the property $\left(P^{*}\right)$ but it does not satisfy any of the conditions of Lemma 3. This case corresponds to the functions $F(t)=\tan \left(\frac{\pi t}{2}\right)$ and $G(t)=t$ for all $t \in(0,1]$ in Proposition 4 .

\section{Fixed-Point Theory in the Setting of Non-Archimedean Fuzzy Metric Spaces}

In this section, we introduce the main results of this work in the setting of nonArchimedean fuzzy metric spaces. The main advantages are based on the fact that we do not assume that any of the auxiliary functions involved in the contraction conditions are monotone. This forces us to do some additional work in order to control the behavior of the sequences involved in the proofs. For instance, in the following lemma, we introduce a new condition on the non-Archimedean fuzzy metric space (in the sense of George and Veeramani) in order to guarantee that the sequences involved in the proofs of fixed-point theorems satisfy additional properties, which are of great importance henceforth. 
Lemma 4. Each non-Archimedean fuzzy metric space (in the sense of George and Veeramani) $(X, M, *)$ whose t-norm $*$ is continuous at the 1-boundary satisfies the following property (called $\left.\mathcal{N C}^{*}\right):$ if $\left\{x_{n}\right\} \subseteq X$ is a sequence which is not $M$-Cauchy and it verifies

$$
\lim _{n \rightarrow+\infty} M\left(x_{n}, x_{n+1}, t\right)=1 \quad \text { for all } t>0,
$$

then there exists $\varepsilon_{0} \in(0,1)$ and $t_{0}>0$, and two partial subsequences $\left\{x_{m_{k}}\right\}$ and $\left\{x_{n_{k}}\right\}$ of $\left\{x_{n}\right\}$ such that

$$
\begin{aligned}
& k<m_{k}<n_{k}<m_{k+1} \quad \text { and } \\
& M\left(x_{m_{k}}, x_{n_{k}-1}, t_{0}\right) \geq 1-\varepsilon_{0}>M\left(x_{m_{k}}, x_{n_{k}}, t_{0}\right)
\end{aligned}
$$

for all $k \in \mathbb{N}$, and also

$$
\lim _{k \rightarrow+\infty} M\left(x_{m_{k}}, x_{n_{k}}, t_{0}\right)=\lim _{k \rightarrow+\infty} M\left(x_{m_{k}-1}, x_{n_{k}-1}, t_{0}\right)=1-\varepsilon_{0}
$$

Proof. It follows by applying the same arguments given in Theorem 22 of [29].

Notice that the main difference between Definition 5 and Lemma 4 is the placement of the inequalities (1) and (3), which plays an important role henceforth (in order to guarantee that some sequences converge from the left). Additionally, notice that in the following statements, we do not assume any kind of monotonicity on the auxiliary functions $\varphi$ and $\eta$.

Theorem 3. Let $(X, M, *)$ be an $M$-complete non-Archimedean fuzzy metric space whose t-norm is continuous at the 1-boundary, and let $\varphi, \eta:(0,1] \rightarrow \mathbb{R}$ be two functions such that $(\varphi, \eta) \in \mathcal{F}$. Let $T: X \rightarrow X$ be self-mapping, satisfying the following contractivity condition:

$$
\varphi(M(T u, T v, s)) \geq \eta(M(u, v, s)) \quad \text { for all } u, v \in X \text { with } T u \neq T v \text { and all } s>0 .
$$

Then, each iterative Picard sequence $\left\{T^{n} u_{0}\right\}$ converges to the unique fixed point of $T$ for every initial condition of $u_{0} \in X$.

Proof. To show the existence of the fixed point of $T$, let us start with an arbitrary point $u_{0} \in X$. We define the sequence $\left\{u_{n}\right\}$ by $T^{n} u_{0}=u_{n}$ for all $n \in \mathbb{N} \cup\{0\}$ and denote $d_{n}(s)=M\left(u_{n}, u_{n+1}, s\right)$ for all $n \in \mathbb{N} \cup\{0\}$ and all $s>0$. Indeed, if there exists $n_{0} \in \mathbb{N}$ such that $u_{n_{0}+1}=u_{n_{0}}$, then $u_{n_{0}}$ is a fixed point of $T$. So, we next suppose that $u_{n} \neq u_{n+1}$ for all $n \in \mathbb{N} \cup\{0\}$, which means that the following holds:

$$
T u_{n} \neq T u_{n+1} \quad \text { for all } n \in \mathbb{N} \cup\{0\}
$$

Taking $u=u_{n}$ and $v=u_{n+1}$ in the contractivity condition (4), we deduce that for all $n \in \mathbb{N} \cup\{0\}$ and all $s>0$, the following holds:

$$
\varphi\left(M\left(u_{n+1}, u_{n+2}, s\right)\right)=\varphi\left(M\left(T u_{n}, T u_{n+1}, s\right)\right) \geq \eta\left(M\left(u_{n}, u_{n+1}, s\right)\right) .
$$

First, we aim to prove that the sequence $\left\{d_{n}(s)\right\}_{n \in \mathbb{N}}$ is nondecreasing. Let $s>0$ be arbitrary. We consider two cases depending on whether $M\left(u_{n}, u_{n+1}, s\right)=1$ or $M\left(u_{n}, u_{n+1}, s\right)<$ 1.

Case 1. If $M\left(u_{n}, u_{n+1}, s\right)=1$, then we have the following:

$$
\varphi\left(M\left(T u_{n}, T u_{n+1}, s\right)\right) \geq \eta\left(M\left(u_{n}, u_{n+1}, s\right)\right)=\eta(1) .
$$


In such a case, condition $\left(C_{3}\right)$ leads to $M\left(u_{n+1}, u_{n+2}, s\right)=M\left(u_{n}, u_{n+1}, s\right)=1$. So, we can say that $d_{n+1}(s) \geq d_{n}(s)$.

Case 2. If $M\left(u_{n}, u_{n+1}, s\right)<1$, from condition (C1) and (4), we can deduce the following:

$$
M\left(u_{n+1}, u_{n+2}, s\right)>M\left(u_{n}, u_{n+1}, s\right),
$$

which means that $d_{n+1}(s)>d_{n}(s)$. Hence, the sequence $\left\{d_{n}(s)\right\}_{n \in \mathbb{N}}$ is nondecreasing, and one can find $d(s) \in(0,1]$ such that $d_{n}(s) \rightarrow d(s)$, as $n \rightarrow+\infty$, for all $s>0$.

Next, we prove that $d(s)=1$ for all $s>0$. Let $s>0$ be arbitrary. If there exists $n_{0} \in \mathbb{N}$ such that $d_{n_{0}}(s)=1$, then $d_{n_{0}+1}(s) \geq d_{n_{0}}(s)=1$, so $d_{n_{0}+1}(s)=1$. In this case, we have that $d_{n}(s)=1$ for all $n \geq n_{0}$, which implies that $d(s)=\lim _{n \rightarrow \infty} d_{n}(s)=1$. Next, we suppose the following:

$$
d_{n}(s)=M\left(u_{n}, u_{n+1}, s\right)<1 \quad \text { for all } n \in \mathbb{N} .
$$

In this case, by (4) and condition $\left(C_{1}\right)$, we have the following:

$$
\varphi\left(d_{n+1}(s)\right) \geq \eta\left(d_{n}(s)\right)>\varphi\left(d_{n}(s)\right),
$$

and

$$
d_{n+1}(s)>d_{n}(s) \text { for all } n \in \mathbb{N} \text {. }
$$

In order to prove $d(s)=1$, suppose on the contrary that $d(s)<1$. In such a case, the following holds:

$$
0<d_{n}(s)<d_{n+1}(s)<d(s)<1 \text { for all } n \in \mathbb{N} .
$$

Taking into account that

$$
\lim _{n \rightarrow \infty} d_{n+1}(s)=\lim _{n \rightarrow \infty} d_{n}(s)=d(s),
$$

it follows that

$$
\lim _{n \rightarrow \infty} \varphi\left(d_{n+1}(s)\right)=\lim _{n \rightarrow \infty} \varphi\left(d_{n}(s)\right)=\lim _{r \rightarrow d(s)^{-}} \varphi(r) .
$$

Taking limits in (5) as $n \rightarrow \infty$, we have the following:

$$
\lim _{n \rightarrow \infty} \eta\left(d_{n}(s)\right)=\lim _{r \rightarrow d(s)^{-}} \varphi(r) .
$$

However, this contradicts the condition $\left(C_{2}\right)$ because the following is true:

$$
\begin{aligned}
\lim _{r \rightarrow d(s)^{-}} \varphi(r) & =\lim _{n \rightarrow \infty} \eta\left(d_{n}(s)\right) \\
& =\eta\left(d(s)^{-}\right) \\
& >\limsup _{r \rightarrow d(s)^{-}} \varphi(r) \\
& \geq \lim _{r \rightarrow d(s)^{-}} \varphi(r) .
\end{aligned}
$$

Hence, $d(s)=1$ for all $s>0$. From Proposition 3, we can claim that the sequence $\left\{u_{n}\right\}$ is either almost periodic or infinite. For the first case, one can easily deduce that the sequence $\left\{u_{n}\right\}$ is almost constant, that is, there exists $n_{0} \in \mathbb{N}$ and $u^{*} \in X$ such that $u_{n}=u^{*}$ for all $n \geq n_{0}$. In this case, $u^{*}$ is a fixed point of $T$, and the part of the proof about the existence of the fixed point of $T$ is completed. So, we suppose that $u_{n_{1}} \neq u_{n_{2}}$ for any $n_{1}, n_{2} \in \mathbb{N}$ such that $n_{1} \neq n_{2}$ (that is $\left\{u_{n}\right\}$ is an infinite sequence). In this case, we have the following:

$$
T u_{n_{1}} \neq T u_{n_{2}} \quad \text { for any } n_{1}, n_{2} \in \mathbb{N} \text { such that } n_{1} \neq n_{2} \text {. }
$$


We continue the proof in the latter case, where we keep in mind that (6) holds. Next, we aim to prove that $\left\{u_{n}\right\}$ is a $M$-Cauchy sequence. Suppose that $\left\{u_{n}\right\}$ is not an $M$-Cauchy sequence. According to Lemma 4 , one can find $L_{0} \in(0,1)$ and $s_{0}>0$ and two partial subsequences $\left\{u_{m_{k}}\right\}$ and $\left\{u_{n_{k}}\right\}$ of $\left\{u_{n}\right\}$ such that for all $k \in \mathbb{N}$,

$$
\begin{aligned}
& k<m_{k}<n_{k}<m_{k+1} \text { and } \\
& M\left(u_{m_{k}}, u_{n_{k}-1}, s_{0}\right) \geq L_{0}>M\left(u_{m_{k}}, u_{n_{k}}, s_{0}\right)
\end{aligned}
$$

and also

$$
\lim _{k \rightarrow+\infty} M\left(u_{m_{k}}, u_{n_{k}}, s_{0}\right)=\lim _{k \rightarrow+\infty} M\left(u_{m_{k}-1}, u_{n_{k}-1}, s_{0}\right)=L_{0} .
$$

Since $\lim _{k \rightarrow+\infty} M\left(u_{m_{k}-1}, u_{n_{k}-1}, s_{0}\right)=L_{0}<1$, there exists $K_{0} \in \mathbb{N}$ such that the following holds:

$$
M\left(u_{m_{k}-1}, u_{n_{k}-1}, s_{0}\right)<1 \quad \text { for all } k \geq K_{0} .
$$

Therefore, from (4), for all $k \geq K_{0}$, we obtain

$$
\varphi\left(M\left(u_{m_{k}}, u_{n_{k}}, s_{0}\right)\right)=\varphi\left(M\left(T u_{m_{k}-1}, T u_{n_{k}-1}, s_{0}\right)\right) \geq \eta\left(M\left(u_{m_{k}-1}, u_{n_{k}-1}, s_{0}\right)\right),
$$

which implies the following:

$$
M\left(u_{m_{k}}, u_{n_{k}}, s_{0}\right)>M\left(u_{m_{k}-1}, u_{n_{k}-1}, s_{0}\right) \quad \text { for all } k \geq K_{0} .
$$

This means, by (7), that

$$
M\left(u_{m_{k}-1}, u_{n_{k}-1}, s_{0}\right)<M\left(u_{m_{k}}, u_{n_{k}}, s_{0}\right)<L_{0}<1 \quad \text { for all } k \geq K_{0} .
$$

Then it follows from (8) that $M\left(u_{m_{k}-1}, u_{n_{k}-1}, s_{0}\right) \rightarrow L_{0}^{-}$and $M\left(u_{m_{k}}, u_{n_{k}}, s_{0}\right) \rightarrow L_{0}^{-}$. Taking the limit superior in (9), we obtain the following:

$$
\begin{aligned}
\eta\left(L_{0}^{-}\right) & =\lim _{k \rightarrow \infty} \eta\left(M\left(u_{m_{k}-1}, u_{n_{k}-1}, s_{0}\right)\right) \\
& \leq \limsup _{k \rightarrow \infty} \varphi\left(M\left(u_{m_{k}}, u_{n_{k}}, s_{0}\right)\right) \\
& \leq \limsup _{s \rightarrow L_{0}^{-}} \varphi(s)
\end{aligned}
$$

which contradicts the condition $\left(C_{2}\right)$. Thus $\left\{u_{n}\right\}$ is an $M$-Cauchy sequence. As $(X, M, *)$ is $M$-complete, then there exists $u^{*} \in X$ such that $\left\{u_{n}\right\} M$-converges to $u^{*}$, that is,

$$
\lim _{n \rightarrow+\infty} M\left(u_{n}, u^{*}, s\right)=1 \quad \text { for all } s>0 .
$$

To prove that $u^{*}$ is a fixed point of $T$, assume, by contradiction, that $u^{*} \neq T u^{*}$. As the sequence $\left\{u_{n}\right\}$ is infinite, then there exists $n_{0} \in \mathbb{N}$ such that $u_{n} \neq u^{*}$ and $u_{n} \neq T u^{*}$ for all $n \geq n_{0}$. From (4), we have the following:

$$
\varphi\left(M\left(u_{n+1}, T u^{*}, s\right)\right)=\varphi\left(M\left(T u_{n}, T u^{*}, s\right)\right) \geq \eta\left(M\left(u_{n}, u^{*}, s\right)\right)
$$

for all $n \geq n_{0}$ and $s>0$. To prove that $M\left(u_{n+1}, T u^{*}, s\right) \geq M\left(u_{n}, u^{*}, s\right)$, we have two cases. Case 1. If $M\left(u_{n}, u^{*}, s\right)=1$, then

$$
\varphi\left(M\left(u_{n+1}, T u^{*}, s\right)\right) \geq \eta\left(M\left(u_{n}, u^{*}, s\right)\right)=\eta(1) .
$$

In such a case, condition $\left(C_{3}\right)$ guarantees that $M\left(u_{n+1}, T u^{*}, s\right)=M\left(u_{n}, u^{*}, s\right)=1$. We can say $M\left(u_{n+1}, T u^{*}, s\right) \geq M\left(u_{n}, u^{*}, s\right)=1$.

Case 2. If $M\left(u_{n}, u^{*}, s\right)<1$, from (4), we have the following:

$$
M\left(u_{n+1}, T u^{*}, s\right)>M\left(u_{n}, u^{*}, s\right) .
$$


In both cases, we have that

$$
1 \geq M\left(u_{n+1}, T u^{*}, s\right) \geq M\left(u_{n}, u^{*}, s\right) \quad \text { for all } n \in \mathbb{N} \text { and all } s>0 \text {. }
$$

By (9), we conclude that

$$
\lim _{n \rightarrow+\infty} M\left(T u_{n}, T u^{*}, s\right)=1 \quad \text { for all } s>0,
$$

which means that the sequence $\left\{u_{n}\right\}$ is $M$-convergent and it converges to $T u^{*}$. The uniqueness of the limit of a convergent sequence in a fuzzy metric space shows that $T u^{*}=u^{*}$. To check the uniqueness of the fixed point, we assume that $u^{*}, u^{\prime} \in X$ are two distinct fixed points of $T$. Since $T u^{*} \neq T u^{\prime}$, then for all $s>0$, we have

$$
\varphi\left(M\left(T u^{*}, T u^{\prime}, s\right)\right) \geq \eta\left(M\left(u^{*}, u^{\prime}, s\right)\right),
$$

which together with condition $\left(C_{1}\right)$, implies that

$$
M\left(T u^{*}, T u^{\prime}, s\right)>M\left(u^{*}, u^{\prime}, s\right) \text { for all } s>0 .
$$

If we suppose that $M\left(u^{*}, u^{\prime}, s_{0}\right)<1$ for some $s_{0}>0$, then we have the following:

$$
\begin{aligned}
\varphi\left(M\left(u^{*}, u^{\prime}, s_{0}\right)\right) & =\varphi\left(M\left(T u^{*}, T u^{\prime}, s_{0}\right)\right) \\
& \geq \eta\left(M\left(u^{*}, u^{\prime}, s_{0}\right)\right) \\
& >\varphi\left(M\left(u^{*}, u^{\prime}, s_{0}\right)\right)
\end{aligned}
$$

which is a contradiction. Hence, $M\left(u^{*}, u^{\prime}, s\right)=1$ for all $s>0$, but this contradicts that $u^{*}$ and $u^{\prime}$ are distinct. Therefore, the mapping $T$ has a unique fixed point.

Remark 1. From Axiom $\left(F_{2}\right)$, we can easily find the possible case in which there exist two distinct points $u, v \in X$ satisfying $M\left(u, v, s_{0}\right)=1$ for some $s_{0} \in(0,+\infty)$. In such a case, we deduce from Lemma 1 that $M(u, v, s)=1$ for all $s \in\left[s_{0},+\infty\right)$, which shows that the contractivity condition stated in (2) includes the case that $\varphi(t) \geq \eta(1)$ for some $t \in(0,1]$. Thus, the conclusion of Theorem 3 remains valid by removing the condition $T u \neq T v$ on the contraction constraint in inequality (2), which is stated as the following corollary.

Corollary 3. Let $(X, M, *)$ be an $M$-complete non-Archimedean fuzzy metric space under a continuous $t$-norm $*$ at the 1-boundary, and let $\varphi$ and $\eta$ be two mappings such that $(\varphi, \eta) \in \mathcal{F}$. Additionally, consider a mapping $T: X \rightarrow X$ satisfying the following contractive condition:

$$
\varphi(M(T u, T v, s)) \geq \eta(M(u, v, s)) \quad \text { for all } u, v \in X \text { and all } s>0 .
$$

Then, each iterative Picard sequence $\left\{T^{n} u_{0}\right\}$ converges to the unique fixed point of $T$ for every initial condition of $u_{0} \in X$.

In the following result, we involve the property $\left(P^{*}\right)$.

Theorem 4. Let $(X, M, *)$ be an $M$-complete non-Archimedean fuzzy metric space whose t-norm is continuous at the 1-boundary, and let $\varphi, \eta:(0,1] \rightarrow \mathbb{R}$ be two functions such that $\varphi$ is left-continuous on $(0,1)$ and they satisfy the following conditions:

$\left(C_{1}\right)$ For every $1>r \geq t>0$, one has $\eta(r)>\varphi(t)$;

$\left(C_{2}^{\prime}\right) \lim _{s \rightarrow L^{-}} \inf (\eta(s)-\varphi(s))>0 \quad$ for any $L \in(0,1)$;

$\left(C_{3}\right)$ If $t \in(0,1]$ is such that $\varphi(t) \geq \eta(1)$, then $t=1$;

$\left(C_{4}\right)$ At least one of the functions of the pair of $(\varphi, \eta)$ satisfies the property $\left(P^{*}\right)$. 
Let us also consider a mapping $T: X \rightarrow X$ satisfying the following contractive condition:

$$
\varphi(M(T u, T v, s)) \geq \eta(M(u, v, s)) \quad \text { for all } u, v \in X \text { with } T u \neq T v \text { and all } s>0 .
$$

Then, each iterative Picard sequence $\left\{T^{n} u_{0}\right\}$ converges to the unique fixed point of $T$ for every initial condition of $u_{0} \in X$.

Proof. Let $u_{0} \in X$ be an arbitrary point. We define the sequence $\left\{u_{n}\right\}$ by $T^{n} u_{0}=u_{n}$ for all $n \in \mathbb{N} \cup\{0\}$ and we denote $d_{n}(s)=M\left(u_{n}, u_{n+1}, s\right)$ for all $n \in \mathbb{N} \cup\{0\}$ and all $s>0$. A similar analysis to that given in the proof of Theorem 3 shows that the sequence $\left\{d_{n}(s)\right\}$ is nondecreasing and converges to $d(s) \leq 1$ as $n \rightarrow \infty$ for all $s>0$.

Next, we show that $d(s)=1$. By (4), we obtain that

$$
\varphi\left(d_{n}(s)\right) \geq \eta\left(d_{n-1}(s)\right) \quad \text { for all } n \geq 1 \text { and all } s>0 .
$$

From the above inequality, we obtain the following:

$$
\varphi\left(d_{n}(s)\right)-\varphi\left(d_{n-1}(s)\right) \geq \eta\left(d_{n-1}(s)\right)-\varphi\left(d_{n-1}(s)\right) \quad \text { for every } n \geq 1 \text {. }
$$

Therefore,

$$
\sum_{k=1}^{n}\left(\varphi\left(d_{k}(s)\right)-\varphi\left(d_{k-1}(s)\right)\right) \geq \sum_{k=1}^{n}\left(\eta\left(d_{k-1}(s)\right)-\varphi\left(d_{k-1}(s)\right)\right) .
$$

So,

$$
\varphi\left(d_{n}(s)\right) \geq \varphi\left(d_{0}(s)\right)+\sum_{k=1}^{n}\left(\eta\left(d_{k-1}(s)\right)-\varphi\left(d_{k-1}(s)\right)\right) \rightarrow+\infty .
$$

It follows that $\varphi\left(d_{n}(s)\right) \rightarrow+\infty$. At the same time, since $d_{n-1}(s)<d_{n}(s)$, we deduce from condition $\left(C_{1}\right)$ that $\varphi\left(d_{n-1}(s)\right)<\eta\left(d_{n}(s)\right)$ for all $n \in \mathbb{N} \cup\{0\}$ and $s>0$, so $\lim _{n \rightarrow \infty} \eta\left(d_{n}(s)\right)=+\infty$. Therefore, by property $\left(P^{*}\right)$, we have that

$$
\lim _{n \rightarrow \infty} d_{n}(s)=1 \quad \text { for all } s>0
$$

Likewise, we also claim that the sequence $\left\{u_{n}\right\}$ is either almost periodic or infinite, and in this last case, the following holds:

$$
T u_{n_{1}} \neq T u_{n_{2}} \text { for any } n_{1}, n_{2} \in \mathbb{N} \text { such that } n_{1} \neq n_{2} \text {. }
$$

Now we claim that $\left\{u_{n}\right\}$ is a $M$-Cauchy sequence. Suppose that $\left\{u_{n}\right\}$ is not an $M$ Cauchy sequence. According to Lemma 4, one can find $L_{0} \in(0,1), s_{0}>0$ and two partial subsequences $\left\{u_{m_{k}}\right\}$ and $\left\{u_{n_{k}}\right\}$ of $\left\{u_{n}\right\}$ such that for all $k \in \mathbb{N}$, the following holds:

$$
\begin{aligned}
& k<m_{k}<n_{k}<m_{k+1} \text { and } \\
& M\left(u_{m_{k}}, u_{n_{k}-1}, s_{0}\right) \geq L_{0}>M\left(u_{m_{k}}, u_{n_{k}}, s_{0}\right)
\end{aligned}
$$

and also

$$
\lim _{k \rightarrow+\infty} M\left(u_{m_{k}}, u_{n_{k}}, s_{0}\right)=\lim _{k \rightarrow+\infty} M\left(u_{m_{k}-1}, u_{n_{k}-1}, s_{0}\right)=L_{0}
$$
holds:

Since $\lim _{k \rightarrow+\infty} M\left(u_{m_{k}-1}, u_{n_{k}-1}, s_{0}\right)=L_{0}<1$, there exists $K_{0} \in \mathbb{N}$ such that the following

$$
M\left(u_{m_{k}-1}, u_{n_{k}-1}, s_{0}\right)<1 \quad \text { for all } k \geq K_{0} .
$$

Therefore, from (4), for all $k \geq K_{0}$, we obtain the following:

$$
\varphi\left(M\left(u_{m_{k}}, u_{n_{k}}, s_{0}\right)\right)=\varphi\left(M\left(T u_{m_{k}-1}, T u_{n_{k}-1}, s_{0}\right)\right) \geq \eta\left(M\left(u_{m_{k}-1}, u_{n_{k}-1}, s_{0}\right)\right)
$$


which implies the following:

$$
M\left(u_{m_{k}}, u_{n_{k}}, s_{0}\right)>M\left(u_{m_{k}-1}, u_{n_{k}-1}, s_{0}\right) \quad \text { for all } k \geq K_{0} .
$$

This means, by (10), that

$$
M\left(u_{m_{k}-1}, u_{n_{k}-1}, s_{0}\right)<M\left(u_{m_{k}}, u_{n_{k}}, s_{0}\right)<L_{0}<1 \quad \text { for all } k \geq K_{0} .
$$

Then it follows from (10) that $M\left(u_{m_{k}-1}, u_{n_{k}-1}, s_{0}\right) \rightarrow L_{0}^{-}$and $M\left(u_{m_{k}}, u_{n_{k}}, s_{0}\right) \rightarrow L_{0}^{-}$. Since $\varphi$ is left continuous at $L_{0}$, taking limits as $k \rightarrow \infty$ in (11), we have the following:

$$
\begin{aligned}
& \lim _{k \rightarrow \infty}(\eta-\varphi)\left(M\left(u_{m_{k}-1}, u_{n_{k}-1}, s_{0}\right)\right) \\
& \quad \leq \lim _{k \rightarrow \infty}\left[\varphi\left(M\left(u_{m_{k}}, u_{n_{k}}, s_{0}\right)\right)-\varphi\left(M\left(u_{m_{k}-1}, u_{n_{k}-1}, s_{0}\right)\right)\right] \\
& \quad=\varphi\left(L_{0}^{-}\right)-\varphi\left(L_{0}^{-}\right)=0
\end{aligned}
$$

which contradicts the condition $\left(C_{2}\right)$. Thus, $\left\{u_{n}\right\}$ is a $M$-Cauchy sequence. As $(X, M, *)$ is $M$-complete, then there exists $u^{*} \in X$ such that $\left\{u_{n}\right\}$ is $M$-convergent and converges to $u^{*}$, that is,

$$
\lim _{n \rightarrow+\infty} M\left(u_{n}, u^{*}, s\right)=1 \quad \text { for all } s>0 .
$$

The rest of the proof to show that $u^{*}$ is the unique fixed point of $T$ follows as in the proof of Theorem 3.

Corollary 4. Let $(X, M, *)$ be an $M$-complete non-Archimedean fuzzy metric space whose t-norm $*$ is continuous at the 1-boundary and let $T: X \rightarrow X$, satisfying

$$
\varphi(M(T u, T v, s)) \geq \eta(M(u, v, s))), \quad \text { for all } u, v \in X \text { with } T u \neq T v \text { and all } s>0,
$$

where $\varphi, \eta:(0,1] \rightarrow \mathbb{R}$ are two functions satisfying the following conditions:

(i) $\eta$ is nondecreasing;

(ii) $\eta(t)>\varphi(t)$ for every $t \in(0,1)$;

(iii) $\varphi$ is upper semi-continuous from the left;

(iv) If $t \in(0,1]$ is such that $\varphi(t) \geq \eta(1)$, then $t=1$.

Then, each iterative Picard sequence $\left\{T^{n} u_{0}\right\}$ converges to the unique fixed point of $T$ for every initial condition of $u_{0} \in X$.

Proof. It follows from $(i)$ and $(i i)$ that $(\varphi, \eta)$ satisfies the condition $\left(C_{1}\right)$. Let any $L \in(0,1)$. Using (ii) and (iii), we have the following:

$$
\limsup _{s \rightarrow L^{-}} \varphi(s) \leq \varphi(L)<\eta(L)
$$

Since $\eta$ is nondecreasing, then we have $\eta(L) \leq \lim _{s \rightarrow L^{-}} \eta(s)$. Consequently, the condition $\left(C_{2}\right)$ is verified. Therefore, together with condition $(\mathrm{iii})$, the conclusion now follows from Theorem 3.

Here are two examples to illustrate the validity of Theorem 3 and Theorem 4.

Example 5. Let $X=(0, \infty)$ and $t * s=t \cdot s$ be the product $t$-norm. Define the following:

$$
M(x, y, s)=\frac{\min \{x, y\}}{\max \{x, y\}} \quad \text { for all } x, y \in X \text { and } s>0 \text {. }
$$


Apparently, $(X, M, *)$ is a $M$-complete non-Archimedean fuzzy metric space whose $t$-norm is continuous at the 1-boundary. Define $\varphi, \eta:(0,1] \rightarrow \mathbb{R}$ by $\eta(t)=t$ and $\varphi(t)=t^{2}$ for all $t \in(0,1]$, and let $T: X \rightarrow X$,

$$
T x= \begin{cases}\sqrt{x}, & \text { if } x \in(0,1] \\ 1, & \text { if } x \in(1, \infty) .\end{cases}
$$

An easy verification shows that $(\varphi, \eta)$ satisfies all the conditions required in Theorem 3 . It remains to verify that $T$ satisfies the contractivity condition. Let $x, y \in X$ be such that $T x \neq T y$. Then, $x \neq y$, say $x<y$.

Case 1. $0<x<y \leq 1$.

$$
\eta(M(T x, T y, s))=\sqrt{\frac{x}{y}} \geq \frac{x}{y}=\varphi(M(x, y, s)) .
$$

Case 2. $0<x \leq 1<y$.

$$
\eta(M(T x, T y, s))=\sqrt{x} \geq x=\varphi(M(x, y, s)) .
$$

Therefore, the contractivity condition is fulfilled, and T has a unique fixed point (which is 1).

Example 6. Let $X=[0, \infty)$ and $t * s=t \cdot s$ be the product $t$-norm. Define the following:

$$
M(x, y, s)=\exp \left(-\frac{|x-y|}{s}\right) \quad \text { for all } x, y \in X \text { and } s>0 .
$$

It is obvious that $(X, M, *)$ is a M-complete non-Archimedean fuzzy metric space whose $t$-norm is continuous at the 1-boundary. Define $\varphi, \eta:(0,1] \rightarrow \mathbb{R}$ as follows:

$$
\eta(t)=\left\{\begin{array}{ll}
\frac{1}{\ln t^{2}}, & \text { if } 0<t<1, \\
1, & \text { if } t=1,
\end{array} \quad \text { and } \quad \varphi(t)= \begin{cases}\frac{1}{\ln t^{\prime}}, & \text { if } 0<t<1 \\
2, & \text { if } t=1\end{cases}\right.
$$

Let $k \in\left(\frac{1}{2}, \infty\right)$ and let $T: X \rightarrow X$ be Tx $=k x$ for all $x \in X$. The pair $(\varphi, \eta)$ satisfies all the conditions given in Theorem 4 . Let us check that $T$ satisfies the contractivity condition. Let $x, y \in X$ such that $T x \neq T y$. Then, $x \neq y$, say $x<y$.

$$
\eta(M(T x, T y, s))=-\frac{s}{2 k(y-x)} \geq-\frac{s}{y-x}=\varphi(M(x, y, s)) .
$$

Therefore, the contractivity condition is fulfilled, and Thas a unique fixed point (which is 0).

In the following corollary, we highlight that Theorem 2 can be deduced as a consequence of Theorem 3.

Corollary 5. Theorem 2 can be deduced from Theorem 3.

Proof. First of all, it directly follows from properties $\left(p_{1}\right)$ and $\left(p_{2}\right)$ that $(\varphi, \eta)$ satisfies the condition $\left(C_{1}\right)$. Additionally, due to the monotonicity of $\varphi$, we deduce that there exists $\lim _{s \rightarrow L^{-}} \varphi(s)$, which coincides with $\limsup _{s \rightarrow L^{-}} \varphi(s)$ for every $L \in(0,1)$. So, from $\left(p_{3}\right)$, we have for any $L \in(0,1)$ the following:

$$
\eta\left(L^{-}\right) \geq \liminf _{s \rightarrow L^{-}} \eta(s)>\lim _{s \rightarrow L^{-}} \varphi(s)=\limsup _{s \rightarrow L^{-}} \varphi(s) .
$$

Thus, condition $\left(C_{2}\right)$ also holds. Moreover, condition $\left(C_{3}\right)$ follows from property $\left(p_{4}\right)$. Therefore, the conclusion of Theorem 2 can be obtained from Theorem 3. 
Remark 2. In [14], the authors remarked that the conclusion of Theorem 2 remains true while replacing the property $\left(p_{4}\right)$ by $\left(p_{4}^{\prime}\right)$, stated in Corollary 2, [14], as follows:

$$
\left(p_{4}^{\prime}\right): \quad \eta(1) \geq \sup \{\eta(s): s \in(0,1)\} .
$$

Indeed, we can verify that under the property $\left(p_{2}\right)$, the condition $\left(p_{4}^{\prime}\right)$ implies the property $\left(p_{4}\right)$. To prove it, let $t \in(0,1]$ be such that $\varphi(t) \geq \eta(1)$. To check that $t=1$, we assume that $t<1$. From property $\left(p_{4}^{\prime}\right)$, we have the following:

$$
\varphi(t) \geq \eta(1) \geq \sup \{\eta(s): s \in(0,1)\} \geq \eta(t),
$$

which contradicts the property $\left(p_{2}\right)$. Therefore, $t=1$. Hence, $\varphi(1) \geq \eta(1)$. Consequently, the conclusion of Corollary 2 in [14] can also be obtained from Theorem 3.

Corollary 6. Let $(X, d)$ be a complete metric space, let $\varphi, \eta$ be two mappings such that $(\varphi, \eta) \in \mathcal{F}$ and let $\vartheta$ be a nondecreasing and continuous function from $(0, \infty)$ into $(0,1)$ such that $\lim _{t \rightarrow \infty} \vartheta(t)=1$. Let $T: X \rightarrow X$ be a mapping for which there exists $(\varphi, \eta) \in \mathcal{F}$ such that

$$
\varphi\left([\vartheta(s)]^{d(T u, T v)}\right) \geq \eta\left([\vartheta(s)]^{d(u, v)}\right)
$$

for all $u, v \in X$ with $T u \neq T v$ and all $s>0$. Then, each iterative Picard sequence $\left\{T^{n} u_{0}\right\}$ converges to the unique fixed point of $T$ for every initial condition of $u_{0} \in X$.

Proof. As we commented in Example 3, if $*$ is a t-norm such that $* \leq *_{p}$ and we define $M: X \times X \times(0,+\infty) \rightarrow[0,1]$ such that

$$
M(u, v, s)=[\vartheta(s)]^{d(u, v)}
$$

for each $u, v \in X$ and all $s>0$, then $(X, M, *)$ is a non-Archimedean fuzzy metric space. Since $(X, d)$ is complete, so is $(X, M, *)$. Further, the contractivity condition (12) is equivalent to (2). Therefore, Theorem 3 guarantees the validity of the conclusion.

\section{Application}

Let consider the Banach space $C([0, I], \mathbb{R})$ of all continuous functions defined on a real interval $[0, I]$ (where $I>0$ ) endowed with the supremum norm

$$
\|x\|=\sup _{r \in[0,1]}|x(r)| \quad \text { for all } x \in C([0, I], \mathbb{R}),
$$

with the induced complete metric

$$
d(x, y)=\sup _{r \in[0, I]}|x(r)-y(r)| .
$$

On this setting, consider the following integral equation:

$$
x(r)=g(r)+\int_{0}^{r} F(r, s, x(s)) d s, \text { for all } r \in[0, I] .
$$

Additionally, consider the fuzzy metric $M$ with product t-norm as follows:

$$
M(x, y, t)=\frac{t}{t+d(x, y)} \quad \text { for all } x, y \in C([0, I], \mathbb{R}) \text { and all } t>0 .
$$

According to George and Veeramani, the standard fuzzy metric space and the corresponding metric space are endowed by the same topology. So the fuzzy metric space defined by (14) is complete. 
Theorem 5. Let us consider the integral operator $T$ on $C([0, I], \mathbb{R})$ as

$$
T(y(t))=g(t)+\int_{0}^{t} F(r, s, y(s)) d s,
$$

where $f:[0, I] \times[0, I] \longrightarrow[0, \infty)$ is such that $f \in L^{1}([0, I], \mathbb{R})$, for all $x, y \in C([0, I], \mathbb{R})$, $t, s \in[0, I]$, and $F$ satisfies the following condition:

$$
|F(s, t, x(t))-F(s, t, y(t))| \leq f(t, s)|x(s)-y(s)|,
$$

for all $x, y \in C([0, I], \mathbb{R})$ and all $t, s \in[0, I]$, where

$$
\sup _{t \in[0,1]} \int_{0}^{t} f(t, s) d s \leq k<1 .
$$

Then, the integral equation (13) has a unique solution.

Proof. Given $x, y \in C([0, I], \mathbb{R})$ and $t>0$, we have that

$$
\begin{aligned}
|T(x(t))-T(y(t))| & \leq \int_{0}^{r}|F(t, s, x(s))-F(t, s, y(s))| d s \\
& \leq \int_{0}^{t} f(t, s)|x(s)-y(s)| d s \\
& \leq d(x, y) \int_{0}^{t} f(t, s) d s \\
& \leq k d(x, y) .
\end{aligned}
$$

Therefore, the following holds:

$$
d(T x, T y) \leq k d(x, y)
$$

Using (14), we can write

$$
d(T x, T y) \leq k d(x, y) \leq d(x, y),
$$

which can be interpreted as the following:

$$
s+d(T x, T y) \leq s+d(x, y) .
$$

Hence we have

$$
\frac{s}{s+d(T x, T y)} \geq \frac{s}{s+d(x, y)},
$$

which means that the following holds:

$$
M(T x, T y, s) \geq M(x, y, s) \geq M(x, y, s)^{2} .
$$

If we take $\varphi(s)=s$ and $\eta(s)=s^{2}$, then the above inequality can be written as follows:

$$
\varphi(M(T x, T y, s)) \geq \eta(M(x, y, s)) .
$$

Since all the conditions of Theorem 3 hold, we deduce that (13) has a unique solution.

\section{Conclusions}

Inspired by Proinov contractions, very recently, some authors extended his main results to the setting of fuzzy metric spaces (in the sense of George and Veeramani). Although Proinov's assumptions on the auxiliary functions (that play a key role in the 
contractivity condition) are very weak, one of them attracted the attention of the researchers in this field of study: the nondecreasing character of one of the involved functions.

In this paper, we have introduced a first approach in the direction of avoiding the monotonicity condition on the auxiliary functions. Accordingly, we have described a novel family of fuzzy contractions in the set of non-Archimedean fuzzy metric spaces that do not need such a condition to appropriately develop some results about the existence and uniqueness of fixed points. Our results generalize other previous statements in this area. In this sense, the presented conclusions provide a positive partial solution to one of the open problems posed in [14] for deleting or weakening the hypothesis of nondecreasingness on the auxiliary functions.

Future work is needed in this line of research because it seems reasonable to ask for another conditions in order to guarantee the existence of fixed points in a more general framework.

Author Contributions: Conceptualization, M.Z., A.F.R.L.d.H.; formal analysis, M.Z., A.F.R.L.d.H.; investigation, M.Z., N.S., X.L., A.F., A.F.R.L.d.H.; writing—original draft preparation, M.Z., N.S., A.F.; writing-review and editing, M.Z., X.L., A.F.R.L.d.H. All authors have read and agreed to the published version of the manuscript.

Funding: Xiao-lan Liu is partially supported by National Natural Science Foundation of China (Grant No.11872043), Opening Project of Key Laboratory of Higher Education of Sichuan Province for Enterprise Internationalization and Internet of Things (Grant No.2020WYJ01), Sichuan Science and Technology Program (Grant No. 2019YJ0541) and Scientific Research Project of Sichuan University of Science and Engineering (Grant Nos. 2017RCL54, 2019RC42, and 2019RC08), Opening Project of Sichuan Province University Key Laboratory of Bridge Non-destruction Detecting and Engineering Computing (Grant No. 2019QZJ03), Open Fund Project of Artificial Intelligence Key Laboratory of Sichuan Province (Grant No. 2018RYJ02), Zigong Science and Technology Program (Grant No. 2020YGJC03), 2020 Graduate Innovation Project of Sichuan University of Science and Engineering (Grant No. y2020078). A.F. Roldán López de Hierro is grateful to Project of Ministerio de Ciencia e Innovación (Grant No. PID2020-119478GB-I00) and also to Junta de Andalucía of the Andalusian PAIDI (Grant No. FQM-365). A.F. Roldán López de Hierro is grateful to Project of Ministerio de Ciencia eInnovación (Grant No. PID2020-119478GB-I00) , Junta de Andalucía of the Andalusian PAIDI (Grant No. FQM-365) and Program FEDER Andalucía 2014-2020 (Grant No. Project A-FQM170-UGR20).

Institutional Review Board Statement: Not applicable.

Informed Consent Statement: Not applicable.

Data Availability Statement: Not applicable.

Conflicts of Interest: The authors declare that they have no competing interests.

\section{References}

1. Asgari, M.S.; Mousavi, B. Solving a class of nonlinear matrix equations via the coupled fixed point theorem. Appl. Math. Comput. 2015, 259, 364-373. [CrossRef]

2. Berzig, M. Solving a class of matrix equations via the Bhaskar-Lakshmikantham coupled fixed point theorem. Appl. Math. Lett. 2012, 25, 1638-1643. [CrossRef]

3. Saleem, N.; Abbas, M.; Raza, Z. Fixed fuzzy point results of generalized Suzuki type F-contraction mappings in ordered metric spaces. Georgian Math. J. 2020, 27, 307-320. [CrossRef]

4. Saleem, N.; Ali, B.; Abbas, M.; Raza, Z. Fixed points of Suzuki type generalized multivalued mappings in fuzzy metric spaces with applications. Fixed Point Theory Appl. 2015, 2015, 1-18. [CrossRef]

5. Aydi, H.; Nashine, H.K.; Samet, B.; Yazidi, H. Coincidence and common fixed point results in partially ordered cone metric spaces and applications to integral equations. Nonlinear Anal. TMA 2011, 74, 6814-6825. [CrossRef]

6. Harjani, J.; López, B.; Sadarangani, K. Fixed point theorems for mixed monotone operators and applications to integral equations Nonlinear Anal. TMA 2011, 74, 1749-1760.

7. Salimi, P.; Hussain, N.; Shukla, S.; Fathollahi, S.; Radenović, S. Fixed point results for cyclic $\alpha-\psi \varphi$-contractions with application to integral equations. J. Comput. Appl. Math. 2015, 290, 445-458. [CrossRef]

8. Banach, S. Sur les opérations dans les ensembles abstraits et leur application aux équations intégrales. Fund. Math. 1922, 3, 133-181. [CrossRef] 
9. Proinov, P.D. Fixed point theorems for generalized contractive mappings in metric spaces. J. Fixed Point Theory Appl. 2020, $22,21$. [CrossRef]

10. Wardowski, D. Fixed points of a new type of contractive mappings in complete metric spaces. Fixed Point Theory Appl. 2012, 2012, 94. [CrossRef]

11. Jleli, M.; Samet, B. A new generalization of the Banach contraction principle. J. Inequal. Appl. 2014, 38, 1-8. [CrossRef]

12. Skof, F. Theoremi di punto fisso per applicazioni negli spazi metrici. Atti Accad. Sci. Torino Cl. Sci. Fis. Mat. Natur. 1977, 111, 323-329.

13. Karapınar, E.; de la Sen, M.; Fulga, A. A note on the Górnicki-Proinov type contraction. J. Funct. Spaces 2021. [CrossRef]

14. Roldán López de Hierro, A.F.; Fulga, A.; Karapınar, E. Proinov-Type Fixed-Point Results in Non-Archimedean Fuzzy Metric Spaces. Mathematics 2021, 9, 1594. [CrossRef]

15. Olaru, I.M.; Secelean, N.A. A New Approach of Some Contractive Mappings on Metric Spaces. Mathematics 2021, 9, 1433. [CrossRef]

16. Zadeh, L.A. Fuzzy sets. Inf. Control. 1965, 8, 338-353. [CrossRef]

17. Menger, K. Statistical metrics. Proc. Natl. Acad. Sci. USA 1942, 28, 535-537. [CrossRef]

18. Kaleva, O.; Seikkala, S. On fuzzy metric spaces. Fuzzy Sets Syst. 1984, 12, 215-229. [CrossRef]

19. Schweizer, B.; Sklar, A. Probabilistic Metric Spaces; Dover Publications: New York, NY, USA, 2005.

20. Kramosil, I.; Michálek, J. Fuzzy metrics and statistical metric spaces. Kybernetika 1975, 11, 336-344.

21. Roldán, A.; Martínez-Moreno, J.; Roldán, C. Tripled fixed point theorem in fuzzy metric spaces and applications. Fixed Point Theory Appl. 2013. [CrossRef]

22. George A.; Veeramani P. On some results in fuzzy metric spaces. Fuzzy Sets Syst. 1944, 64, 395-399. [CrossRef]

23. Altun, I.; Miheț, D. Ordered non-Archimedean fuzzy metric spaces and some fixed-point results. Fixed Point Theory Appl. 2010, 782680. [CrossRef]

24. Gregori, V.; Sapena, A. On fixed point theorems in fuzzy metric spaces. Fuzzy Sets Syst. 2020, 125, 245-252. [CrossRef]

25. Karapınar, E. Revisiting the Kannan type contractions via interpolation. Adv. Theory Nonlinear Anal. Appl. 2018, 2, 85-87. [CrossRef]

26. Miheț, D. Fuzzy $\psi$-contractive mappings in non-Archimedean fuzzy metric spaces. Fuzzy Sets Syst. 2008, 159, 739-744. [CrossRef]

27. Roldán López de Hierro, A.F.; Sintunavarat, W. Common fixed point theorems in fuzzy metric spaces using the CLRg property. Fuzzy Sets Syst. 2016, 282, 131-142. [CrossRef]

28. Suganthi, M.; Mathuraiveeran, M.J. Common fixed point theorems in M-fuzzy cone metric space. Results Nonlinear Anal. 2021, 4, 33-46. [CrossRef]

29. Roldán López de Hierro, A.F.; Karapınar, E.; Shahzad, N. Fuzzy ample spectrum contractions in (more general than) nonArchimedean fuzzy metric spaces. arXiv 2021, arXiv:2104.09155.

30. Grabiec, M. Fixed points in fuzzy metric spaces. Fuzzy Sets Syst. 1988, 27, 385-389. [CrossRef] 